\title{
A Composite Scheme for Gas Dynamics in Lagrangian Coordinates
}

\author{
Mikhail Shashkov* and Burton Wendroff $\dagger$ \\ Los Alamos National Laboratory, T-7, MS-B284, Los Alamos, New Mexico 87545 \\ E-mail:*misha@t7.lanl.gov, †bbw@lanl.gov
}

Received August 20, 1998; revised December 22, 1998

\begin{abstract}
One cycle of a composite finite difference scheme is defined as several time steps of an oscillatory scheme such as Lax-Wendroff followed by one step of a diffusive scheme such as Lax-Friedrichs. We apply this idea to gas dynamics in Lagrangian coordinates. We show numerical results in two dimensions for Noh's infinite strength shock problem and the Sedov blast wave problem, and for several one-dimensional problems including a Riemann problem with a contact discontinuity. For Noh's problem the composite scheme produces a better result than that obtained with a more conventional Lagrangian code.

Key Words: Lagrangian gas dynamics; composite scheme; Lax-Wendroff; LaxFriedrichs.
\end{abstract}

\section{INTRODUCTION}

For a system of conservation laws $U_{t}=f_{x}(U)$, it is well known that the Lax-Wendroff (LW) finite difference scheme produces oscillations behind shock waves while the LaxFriedrichs (LF) method is excessively diffusive, smearing out the shocks more than is usually acceptable. In [7, 8] it is shown that an effective way to overcome this behavior of the two methods is to compose them. Thus, the composite scheme is defined by global composition of several LW steps followed by one LF step. If we denote by $L_{W}$ the operator defined by the LW scheme, and by $L_{F}$ the operator defined by the LF scheme, then difference operator $S_{k}$ defined by $k-1$ applications of $L_{W}$ followed by one application of $L_{F}$

$$
S_{k}=L_{F} \circ L_{W} \circ L_{W} \circ \cdots \circ L_{W}
$$

defines the composite scheme which we call LWLFk. The operator $S_{k}$ operates from time level $n$ to $n+k, U^{n+k}=S_{k} U^{n}$.

As early as 1948 Courant and Friedrichs [4] suggested the possibility of doing gas dynamics in Lagrangian, that is, moving with the material, as well as Eulerian, fixed in space, 
coordinates. The preponderance of research in numerical methods since then has been in the Eulerian framework, but the Lagrangian approach is also well-represented, for example, in Richtmyer and Morton and many papers in the Journal of Computational Physics ([1-3] are recent examples with references to earlier literature). Courant and Friedrichs were of the opinion that in more than one dimension the Lagrangian equations were too complicated to be of much use and that was certainly true of the computing power of that time. Now, there are several codes in existence for computing multi-dimensional Lagrangian gas dynamics, but there are some problems which are not done well by these codes, although various fixes have been proposed. One such problem is the Noh problem in the plane [9]: initially the gas is cold and all particles are moving toward the origin with constant velocity. The time evolution is an infinite strength circularly symmetric shock propagating outward. The pressure, density, and velocity are constant behind the shock. The difficulty pointed out by Noh is that the typical code suffers an extreme dip in density at the origin with a corresponding overshoot in internal energy, and the error seems to persist as the grid is refined. This can be seen in Figs. 4a and 5. The reason for this is that the standard artificial viscosity operates if there is a compression, but the codes quickly generate a constant velocity and pressure at the origin, so the viscosity has no effect. Noh called this the wall heating problem. In [7] the Noh problem was done in Eulerian coordinates using the composite idea, and although there was a dip at the origin it was considerably less pronounced than that observed by Noh.

This paper is a first attempt to see if the composite idea performs as well in Lagrangian coordinates, not just for the Noh problem but for a suite of test problems. We have found that for the Noh problem the composite scheme works about as well as it does in Eulerian coordinates, that is, there is a density dip but it is much less pronounced than for the standard code.

We have concentrated on two sets of test problems. The first consists of the Noh [9] and Sedov problems in the plane. The Sedov problem has an initially cold gas at rest. At $t=0$ there is a "point" explosion at the origin.

The second set contains a 1D problem with nonuniform initial density, a 1D piston problem with a jump in mesh spacing, and the Lax 1D Riemann problem. Even in Lagrangian coordinates our composite will cause the contact discontinuity that is present in the Lax problem to spread out, just as it would in Eulerian coordinates, even though the interface is at a fixed grid point. However, by using a Riemann solver to get the flux at the interface and thereby never differencing across it we are able to keep the contact sharp.

In addition to the above we have tried the infamous Saltzman piston problem. The data for this problem and the exact solution are one dimensional, but the grid is not. This causes grief for the standard Lagrangian code, producing extreme non-physical grid distortions as described in [5]. A sophisticated method of subzonal masses is proposed in [1] that eliminates this distortion. Our composite gives a result somewhere between these two.

\section{A FRAMEWORK FOR LAGRANGIAN DIFFERENCE EQUATIONS}

The Lagrangian formulation of gas dynamics uses a coordinate system moving with the velocity field of the fluid. The equations have two parts. In the two dimensional Cartesian plane the first part governs the evolution of the hydrodynamic state variables; namely, $\rho=$ density, $\eta=1 / \rho$, the $x$ and $y$ velocity components $u$ and $v$, the total energy density $E=e+.5\left(u^{2}+v^{2}\right)$, and the internal energy density $e$ and pressure $p \equiv p(\rho, e)$, according 
to the differential equations

$$
\begin{aligned}
\rho \eta_{t} & =\left(u_{x}+v_{y}\right), \\
\rho u_{t} & =-p_{x}, \\
\rho v_{t} & =-p_{y} \\
\rho E_{t} & =-(p u)_{x}-(p v)_{y} .
\end{aligned}
$$

The second part defines the moving coordinate system.

$$
\begin{aligned}
& x_{t}=u, \\
& y_{t}=v,
\end{aligned}
$$

together with the initial conditions

$$
\begin{aligned}
& x(a, b, 0)=a, \\
& y(a, b, 0)=b .
\end{aligned}
$$

The point $(x(a, b, t), y(a, b, t))$ is the current position of the fluid which was initially at $(x(a, b, 0), y(a, b, 0)) .(a, b)$ labels the fluid particle. Thus, for example, the density $\rho$ is

$$
\rho=\rho(x, y)=\rho(x(a, b, t), y(a, b, t))=\hat{\rho}(a, b, t) .
$$

However, we will continue to use the original dependent variable names such as $\rho$ rather than $\hat{\rho}$.

The differential equations can perhaps be better understood in an integral formulation. Following $[4,10]$ we can think of the velocity field $(u, v)$ as effecting a coordinate transformation from $(a, b)$ to $(x, y)$ with Jacobian

$$
j=x_{a} y_{b}-x_{b} y_{a}
$$

Now, if $V(t)$ is a region in the plane with boundary $S(t)$, enclosing and moving with the fluid, then for any $f(x, y)$

$$
\int_{V(0)} f(x(a, b, 0), y(a, b, 0)) j d a d b=\int_{V(t)} f(x, y) d x d y .
$$

Therefore, we can apply the integral operator defining the left side of (5) to the left side of the differential equations (2) and apply the integral operator defining the right side of (5) to the right side of the differential equations. Then, for example, for the $u$ equation we can write

$$
\int_{V(0)} \rho j u_{t} d a d b=-\int_{V(t)} p_{x} d x d y
$$

or

$$
\int_{V(0)} \rho j u_{t} d a d b=-\oint_{S(t)} p d y
$$


But $\rho j$ is independent of time, and

$$
\operatorname{mass}(V(0))=\operatorname{mass}(V(t))=\int_{V(0)} \rho j d a d b .
$$

If we set

$$
d m=\rho j d a d b
$$

then

$$
\int_{V(0)} u_{t} d m=-\oint_{S(t)} p d y
$$

For the full set of equations, put

$$
w=(\eta, u, v, E)^{T}
$$

so that the equations have the form

$$
\rho w_{t}=f_{x}+g_{y},
$$

where

$$
\begin{gathered}
f=(u,-p, 0,-p u)^{T}, \\
g=(v, 0,-p,-p v)^{T},
\end{gathered}
$$

and then

$$
\int_{V(0)} w_{t} d m=\oint_{S(t)}(f d y-g d x) .
$$

This will form the basis of our difference equations.

\section{THE LAX-WENDROFF LAX-FRIEDRICHS COMPOSITE}

We present first a Lagrangian modification of the Lax-Friedrichs scheme in its simplest two-step form. At the beginning of each time step, i.e., at $t^{n}=n \Delta t$, we have a computational region formed by a union of quadrilaterals which we call the primary cells. We suppose that these cells are arranged in a logically rectangular grid. The primary points are defined as the geometric centers of the primary cells. The hydrodynamic states are assumed known and constant in each primary cell. We index the primary states and points with half integer index pairs.

The vertices of the primary cells are the nodes of the grid, and we will refer to them as dual points. We index the dual points with integer index pairs. We will need a set of cells containing the dual points in their interiors, and these dual cells can be defined in several ways. The simplest way is just to use the quadrilateral formed by the four primary points associated with each dual point (see Fig. 1). The dual points are supposed known at $t^{n}$, but not the dual states. 


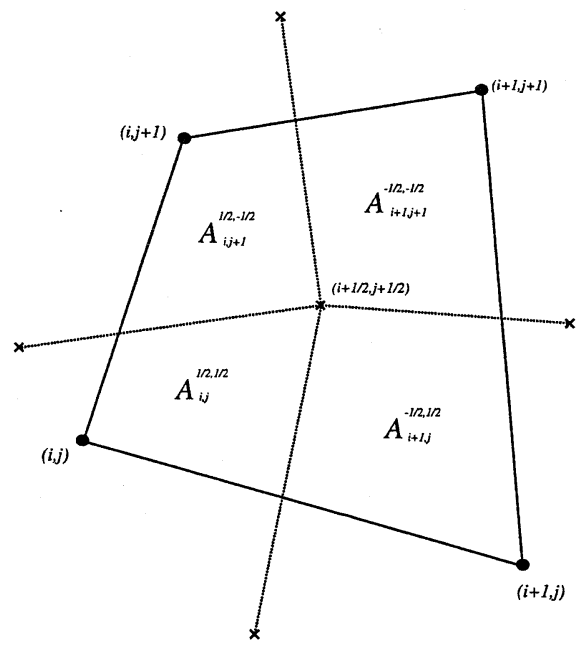

a

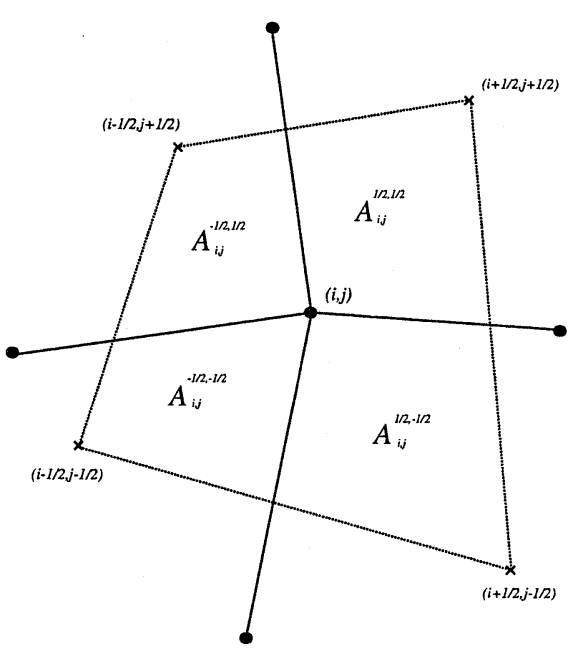

b

FIG. 1. (a) Primary cell. (b) Dual cell.

The mass of each primary cell is the area of the cell times the density. Thus, for the primary cells let

$$
m_{i+1 / 2, j+1 / 2}=\rho_{i+1 / 2, j+1 / 2} A_{i+1 / 2, j+1 / 2},
$$

where

$A_{i+1 / 2, j+1 / 2}=-.5\left[\left(x_{i+1, j}-x_{i, j+1}\right)\left(y_{i, j}-y_{i+1, j+1}\right)+\left(x_{i+1, j+1}-x_{i, j}\right)\left(y_{i+1, j}-y_{i, j+1}\right)\right]$.

The mass of a dual cell is the sum of the masses of its intersection with each of the four neighboring primary cells. If the intersection of the dual cell at $(i, j)$ with the primary cell at $(i+\alpha, j+\beta), \alpha= \pm 1 / 2, \beta= \pm 1 / 2$ has area $A_{i, j}^{\alpha, \beta}$, then the mass of the dual cell is

$$
m_{i, j}=\sum_{\alpha, \beta= \pm 1 / 2} m_{i, j}^{\alpha, \beta}
$$

where

$$
m_{i, j}^{\alpha, \beta}=A_{i, j}^{\alpha, \beta} \rho_{i+\alpha, j+\beta}
$$

The first LF half-step advances the dual states one half time step, from (6) and Fig. 1

$$
w_{i, j}^{n+1 / 2}=\tilde{w}_{i, j}^{n}+\frac{.5 \Delta t}{m_{i, j}} \oint(g d x-f d y)^{n},
$$

where

$$
\tilde{w}_{i, j}^{n}=\left(\sum_{\alpha, \beta= \pm 1 / 2} m_{i, j}^{\alpha, \beta} w_{i+\alpha, j+\beta}^{n}\right) / m_{i, j} .
$$


That is, the dual states at $t^{n}$ are defined as mass-weighted averages of the neighboring primary states, and then advanced a half time step according to the flux contour integral around the edges of the dual cell using primary data at $t^{n}$.

For the line integral, using simple linear interpolation, we have

$$
\begin{aligned}
\oint(f d y)= & \frac{1}{2}\left[\left(f_{i+1 / 2, j-1 / 2}+f_{i+1 / 2, j+1 / 2}\right)\left(y_{i+1 / 2, j-1 / 2}-y_{i+1 / 2, j+1 / 2}\right)\right. \\
& +\left(f_{i+1 / 2, j+1 / 2}+f_{i-1 / 2, j+1 / 2}\right)\left(y_{i+1 / 2, j+1 / 2}-y_{i-1 / 2, j+1 / 2}\right) \\
& +\left(f_{i-1 / 2, j+1 / 2}+f_{i-1 / 2, j-1 / 2}\right)\left(y_{i-1 / 2, j+1 / 2}-y_{i-1 / 2, j-1 / 2}\right) \\
& \left.+\left(f_{i-1 / 2, j-1 / 2}+f_{i+1 / 2, j-1 / 2}\right)\left(y_{i-1 / 2, j-1 / 2}-y_{i+1 / 2, j-1 / 2}\right)\right],
\end{aligned}
$$

and similarly for the $g$ contribution.

The half step is completed by advancing the dual points,

$$
\begin{aligned}
& x_{i, j}^{n+1 / 2}=x_{i, j}^{n}+\frac{\Delta t}{2} u_{i, j}^{*} \\
& y_{i, j}^{n+1 / 2}=y_{i, j}^{n}+\frac{\Delta t}{2} v_{i, j}^{*},
\end{aligned}
$$

where $u^{*}$ and $v^{*}$ are the $x$ and $y$ components of velocity obtained by interpolation from the primary velocity field.

The second half step, from $t^{n+1 / 2}$ to $t^{n+1}$, has several parts. First, the primary states at $t^{n+1 / 2}$ are defined as mass-weighted averages of the neighboring dual states, and then advanced a half time step according to the flux contour integral around the edges of the primary cell using dual data at $t^{n+1 / 2}$. Thus,

$$
w_{i+1 / 2, j+1 / 2}^{n+1}=\tilde{w}_{i+1 / 2, j+1 / 2}^{n+1 / 2}+\frac{.5 \Delta t}{m_{i+1 / 2, j+1 / 2}} \oint(g d x-f d y)^{n+1 / 2},
$$

where

$$
\tilde{w}_{i+1 / 2, j+1 / 2}^{n+1 / 2}=\left(\sum_{\alpha, \beta= \pm 1 / 2} m_{i+1 / 2+\alpha, j+1 / 2+\beta}^{\alpha, \beta} w_{i+1 / 2+\alpha, j+1 / 2+\beta}^{n+1 / 2}\right) / m_{i+1 / 2, j+1 / 2}
$$

However, the specific volume that would be obtained in this way is not used, because the resulting scheme is unstable. Instead, the primary cell volume is computed from the geometry of the advanced dual points. That is, we set

$$
\begin{aligned}
& x_{i, j}^{n+1}=x_{i, j}^{n+1 / 2}+\frac{\Delta t}{2} u_{i, j}^{n+1 / 2}, \\
& y_{i, j}^{n+1}=y_{i, j}^{n+1 / 2}+\frac{\Delta t}{2} v_{i, j}^{n+1 / 2},
\end{aligned}
$$

and then compute the specific volume as the new volume divided by the mass.

The step is completed by placing the primary points in the centers of their cells as defined by the new vertices.

The Lax-Wendroff scheme we will use here is the two-step version proposed by Eilon, Gottlieb, and Zwas for Eulerian coordinates in [6]. The first step is the first LF half step 
described above. The second step is time centered, namely, for the primary points,

$$
w_{i+1 / 2, j+1 / 2}^{n+1}=w_{i+1 / 2, j+1 / 2}^{n}+\frac{\Delta t}{m_{i+1 / 2, j+1 / 2}} \oint(g d x-f d y)^{n+1 / 2},
$$

with the dual data at $t^{n+1 / 2}$ obtained from the LF first half step.

The primary and dual points are obtained exactly as for LF.

Note that this scheme is centered, in two senses. First, all differences are centered and there is no upwinding; and second, all state variables are taken to live at the same point in each cell. This contrasts with the more standard approaches having pressure and density at cell centers and velocities at cell edges or corners.

As stated in the Introduction, the composite consists simply of cycles of $N-1$ timesteps of LW followed by one timestep of LF. In some problems we also did LF on the first and last step.

The Eulerian analogue of the 2D scheme we are using is known not to be optimally stable. The condition we are using is that $\Delta t \max (c / l) \leq .3$, where $c$ is the local sound speed, $l$ is an estimate of the diameter of a cell, and the maximum is taken over the primary cells. However, this is not a mathematically rigorous stability condition, and more investigation of the stability of these methods is needed. In addition, there is another condition that must be satisfied, which is that the volume of any cell be positive. To ensure this we do not allow the volume of a cell to decrease by more than $10 \%$ in any timestep.

\section{NUMERICAL TESTS}

\subsection{The Noh Problem}

The Noh problem is computed at time $t=0.6$, at which time the shock should be at radius 0.2 . The problem is computed in a quarter plane with reflecting boundary conditions on the coordinate axes. The initial grid is uniform and is $50 \times 50$. As stated in the Introduction, initially the density is 1 , the pressure is 0 , and the velocity field is directed toward the center with magnitude 1 . Figure $2 \mathrm{a}$ shows the grid at the final time, and Fig. $2 \mathrm{~b}$ shows a zoomed view. The scheme is LWLF6. Figure 3 is a plot of the density on an axis and on the diagonal.
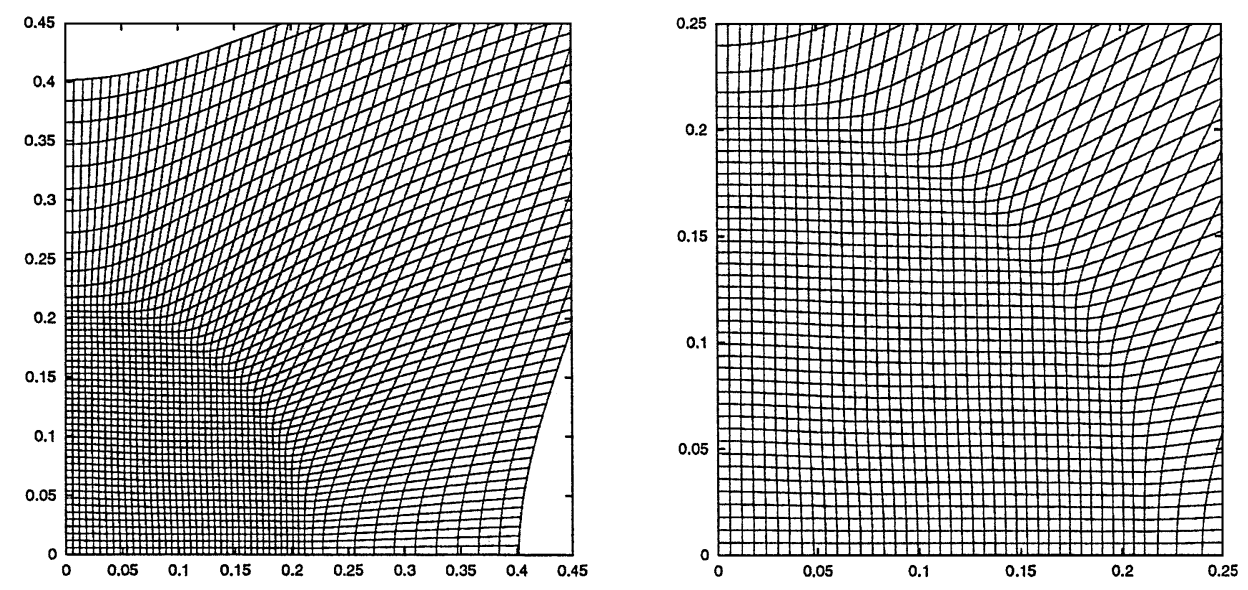

FIG. 2. Noh. (a) Part of the grid at $t=0.2$. (b) Zoom-in on the shocked region. 


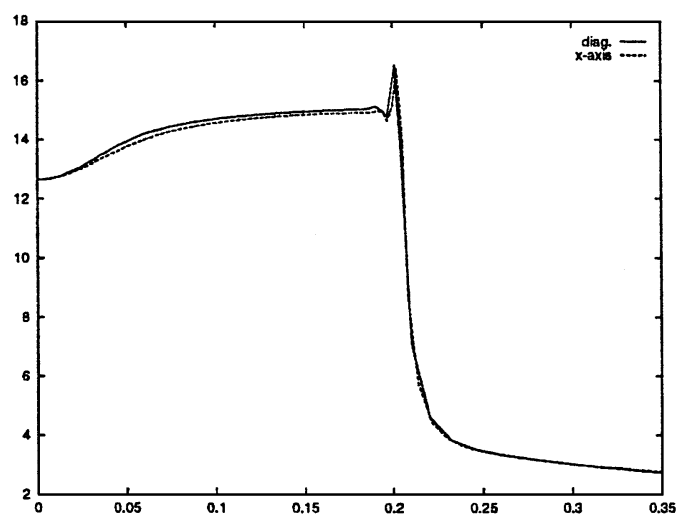

FIG. 3. Noh. Density on an axis and on the diagonal, showing the symmetry.

Figure 4 compares diagonal plots for a standard code and for the composite at successive grid refinements, demonstrating the better behavior of the composite at the center. Figure 5 is a close-up showing the poor convergence of the standard code [3] at the center. Figure 6 is a surface plot of the approximate solution obtained by the composite scheme, which demonstrates good preservation of symmetry.

\subsection{The Sedov Problem}

For this problem the gas is initially cold and at rest with uniform density 1 . There is a "point" explosion at the center at $t=0$.

\subsubsection{Sedov with Cartesian Grid}

As with the Noh problem the basic grid is uniform $50 \times 50$ in the quarter plane. For this grid the cell at the origin of the quarter plane has initial energy 5000. For finer grids the area of the high energy source is kept constant. The scheme is LWLF6. Results are in Figs. 7-9.

\subsubsection{Sedov with Polar Grid}

Here we have computed the Sedov problem on a $25 \times 50$ polar grid with initially equal radial and angular intervals. The central triangles are treated as degenerate quadrilaterals.

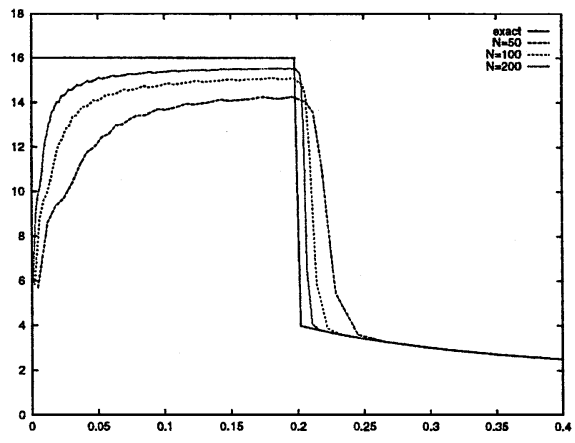

a

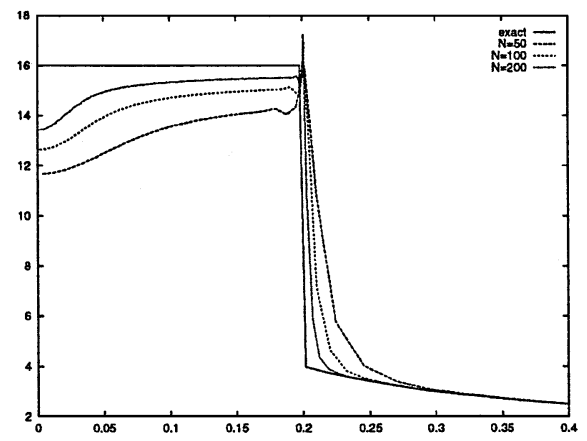

b

FIG. 4. Noh. (a) Standard Lagrangian code. (b) Composite Lagrangian. 


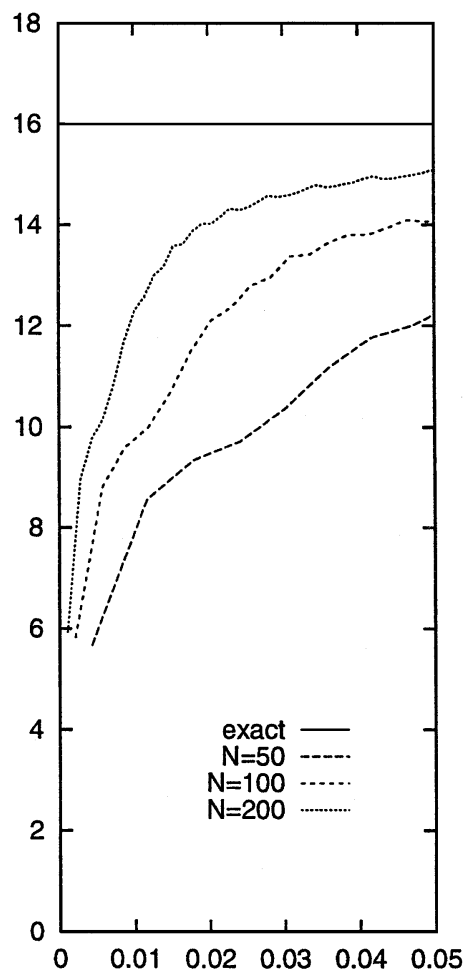

FIG. 5. Noh. Poor convergence of standard code at center.

The initial quarter plane polar grid is generated as follows: for $1 \leq i \leq 26,1 \leq j \leq 51, \Delta \alpha=$ $\pi / 100, \Delta r=L / 50$, where $L=\sqrt{4 / \pi}$ in order to match the energy in the central cells with that of the Cartesian grid. Thus,

$$
\begin{aligned}
& x(i, j)=(j-1) \Delta r \cos \left(\frac{\pi}{2}-(i-1) \Delta \alpha\right) \\
& y(i, j)=(j-1) \Delta r \sin \left(\frac{\pi}{2}-(i-1) \Delta \alpha\right) .
\end{aligned}
$$

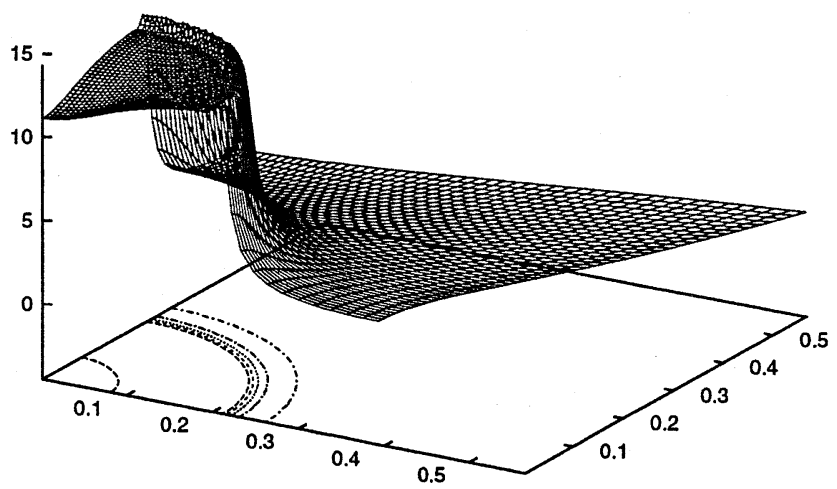

FIG. 6. Noh. Surface plot with contours for composite scheme. 


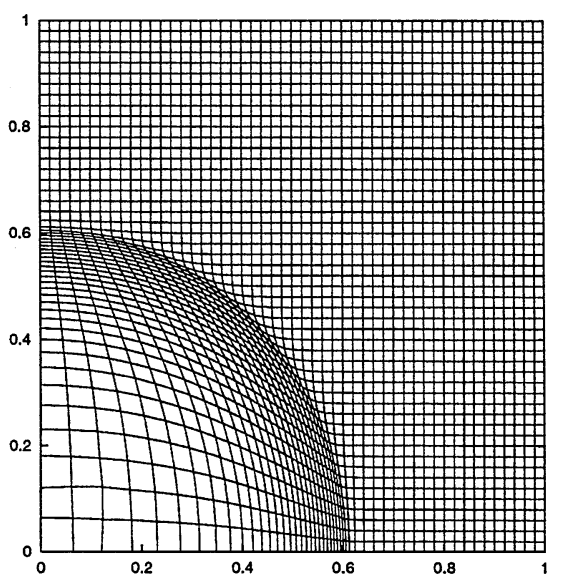

a

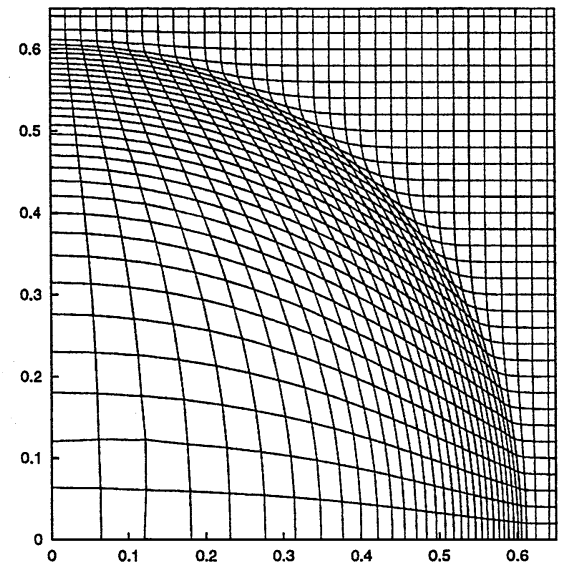

$\mathrm{b}$

FIG. 7. (a) Sedov problem grid at $t=0.1$. (b) Zoom on the shocked region.

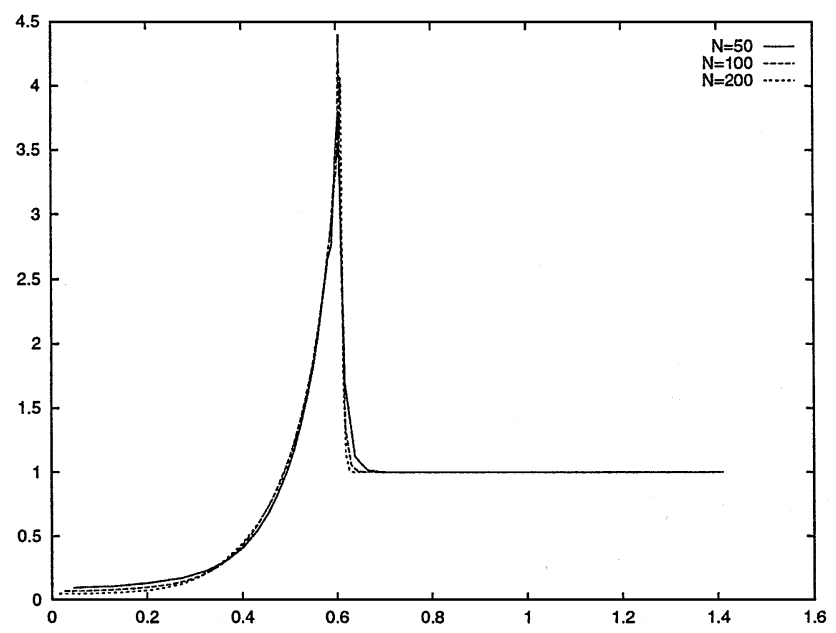

FIG. 8. Sedov density on the diagonal for successive grid refinements.

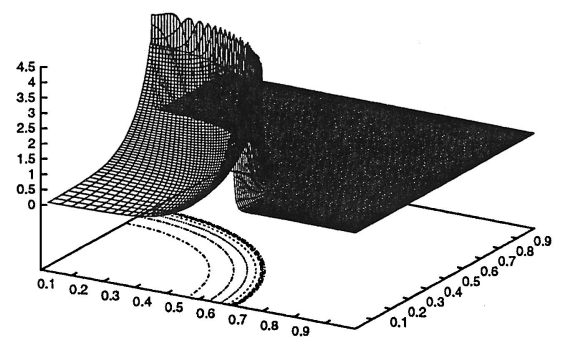

a

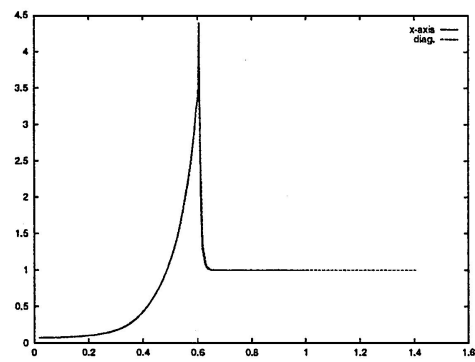

$\mathrm{b}$

FIG. 9. Sedov. (a) Surface plot with contours. (b) Axis and diagonal. 


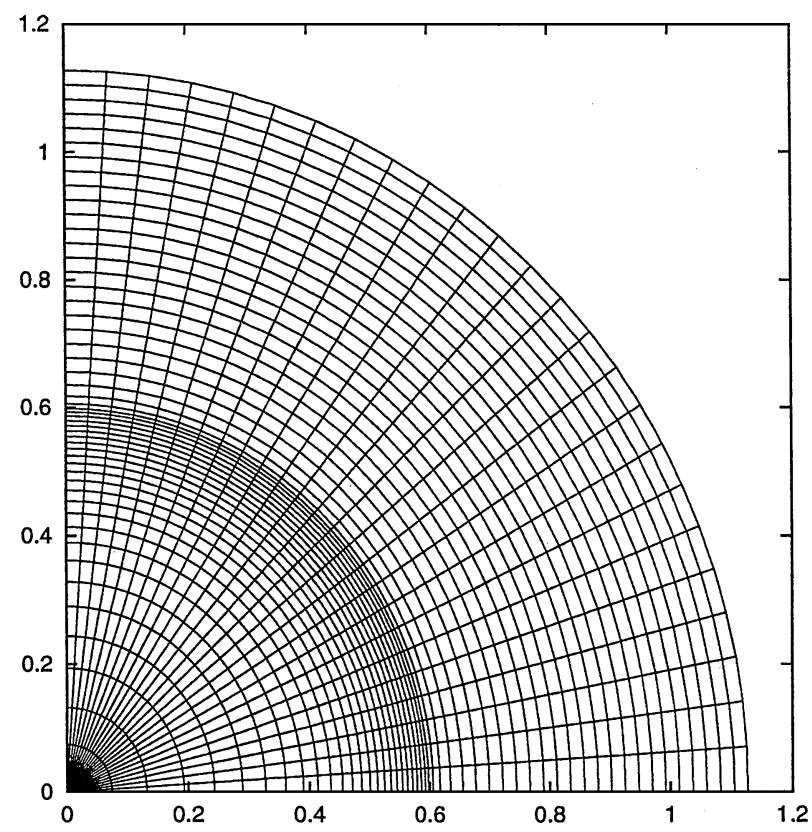

FIG. 10. Sedov (polar). Final grid.

The final grid is in Fig. 10. The density surface and density on the diagonal (all rays have the same values) are in Fig. 11. The scheme is LWLF10.

\subsection{A Modified Saltzman Problem}

The grid is shown in Fig. 12. This differs slightly from the grid in [5]. We have straightened out the grid along the top and bottom in order to eliminate boundary difficulties. The distortions seen in [5] occur well into the interior of the domain. Initially the gas is at rest with density 1 and zero pressure. The piston at the left end moves with speed 1 . The gas is ideal with $\gamma=5 / 3$. At time $t=0.7$ the shock has not quite reached the right reflecting boundary. Figure 13 shows the final grid and Fig. 14 has the density surface with contours, using LWLF10. The dependence on the vertical variable is not satisfactory, but it is a considerable improvement over [5], although falling far short of the result in [1].

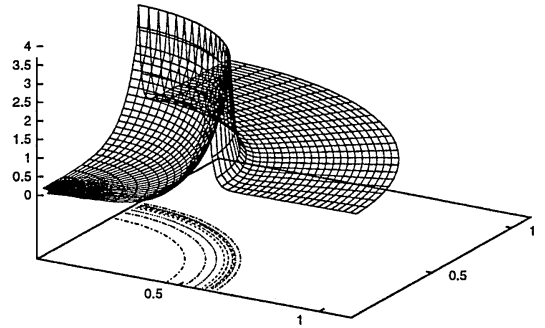

a

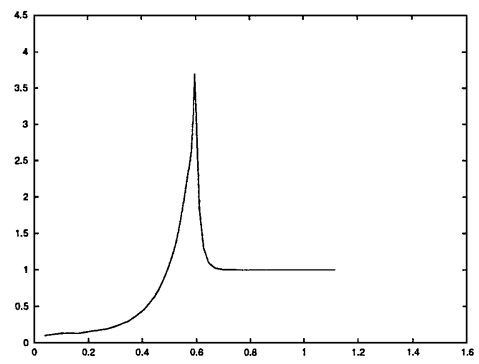

b

FIG. 11. Sedov (polar). (a) Surface plot with contours. (b) Diagonal. 


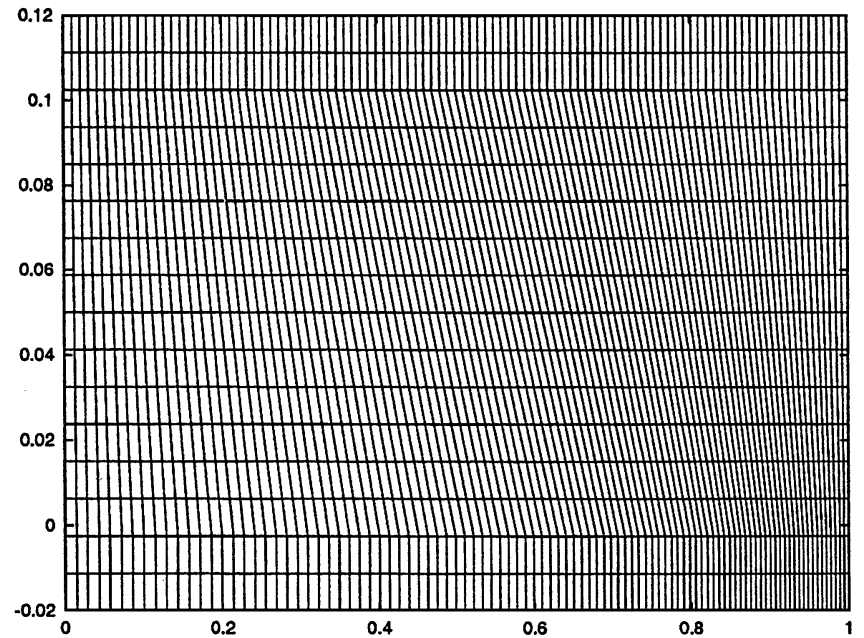

FIG. 12. Saltzman initial grid.

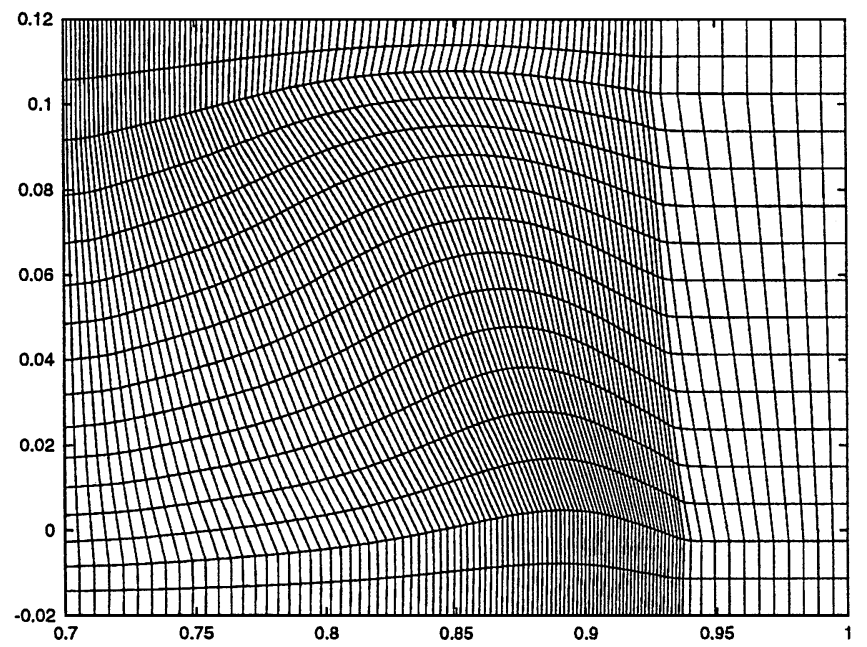

FIG. 13. Saltzman final grid.

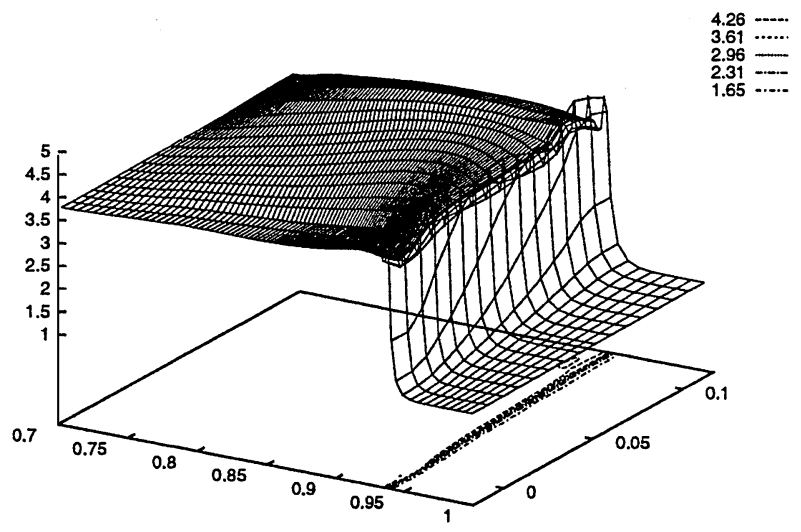

FIG. 14. Saltzman density surface. 


\subsection{The Exponential Background Problem}

This is a $1 \mathrm{D}$ problem in which the initial spatial grid is uniform but the initial hydrodynamic states vary. The exact solution is a shock. The initial data at $t=t_{0}[11]$ are

$$
\begin{aligned}
& u\left(x, t_{0}\right)= \begin{cases}0, & x \geq x_{0}, \\
\frac{\Delta\left(1-\xi_{0}\right)}{t_{0}}, & x \leq x_{0},\end{cases} \\
& \varepsilon\left(x, t_{0}\right)= \begin{cases}0, & x \geq x_{0}, \\
\frac{1}{2}\left(\frac{\Delta}{t_{0}}\right)\left(1+2 \xi_{0}\right), & x \leq x_{0},\end{cases} \\
& \rho\left(x, t_{0}\right)= \begin{cases}\rho_{0} \exp \left[\frac{x-x_{0}}{\Delta}\right], & x \geq x_{0}, \\
3 \rho_{0}\left(1+2 \xi_{0}\right)^{-\frac{5}{2}}, & x \leq x_{0},\end{cases}
\end{aligned}
$$

where $x_{0}$ is the location of the shock front at $t=t_{0}, \xi_{0}=\left(x-x_{0}\right) / \Delta, \Delta$ is characteristic scale of impulse width, and $\rho_{0}$ is the unperturbed background density at $x=x_{0}$.

A self-similar solution for $t>t_{0}$ is given by

$$
\begin{aligned}
& u(x, t)= \begin{cases}0, & x \geq x_{F}(t), \\
\frac{\Delta(1-\xi)}{t}, & x \leq x_{F}(t),\end{cases} \\
& \varepsilon\left(x, t_{0}\right)= \begin{cases}0, & x \geq x_{F}(t), \\
\frac{1}{2}\left(\frac{\Delta}{t}\right)(1+2 \xi), & x \leq x_{F}(t),\end{cases} \\
& \rho\left(x, t_{0}\right)= \begin{cases}\rho_{0} \exp \left[\frac{x-x_{0}}{\Delta}\right], & x \geq x_{F}(t), \\
3 \rho\left(x_{F}\right)(1+2 \xi)^{-\frac{5}{2}}, & x \leq x_{F}(t),\end{cases}
\end{aligned}
$$

where $X_{F}(t)=x_{0}+1.5 \ln \left(t / t_{0}\right)$ is the location of shock front at time $t$ and $\xi=\left(x_{F}(t)-x\right) / \Delta$.

We have used the following values of the parameters: $t_{0}=2, x_{0}=6, \rho_{0}=1, \Delta=4$. This problem has been solved for an ideal gas with $\gamma=2$.

A comparison of the exact and calculated solution is shown in Fig. 15. Figure 15a shows the exact data at the initial time $t=2$ and at the final time $t=6$. Figure $15 \mathrm{~b}$ compares LWLF6 with LWLF10 and the exact solution.

\subsection{Jump Test}

This is a $1 \mathrm{D}$ piston problem testing behavior of the new composite method on a grid with a jump in meshsize. The piston moves with speed 1 . The gas is initially at rest with zero pressure, $\gamma=5 / 3$. The piston creates a shock moving with speed $4 / 3$. The initial grid on the interval $[0,2]$ has fifty intervals of size $2 / 75$ between $x=0$ and $x=4 / 3$ and fifty intervals of size $1 / 75$ from $x=4 / 3$ to $x=2$. In Fig. 16 we compare our algorithm with a more standard code [3]. Note that LW is unstable for this problem, and LF is, as expected, excessively diffusive.

\subsection{The Lax 1D Riemann Problem}

For 1D Riemann problems the point separating the initial states is tracked. Of course, in Lagrangian coordinates that point always has the same label. To avoid smearing the 


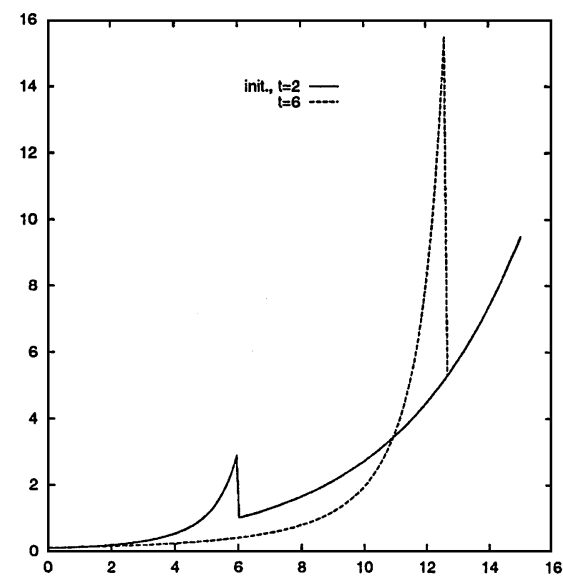

a

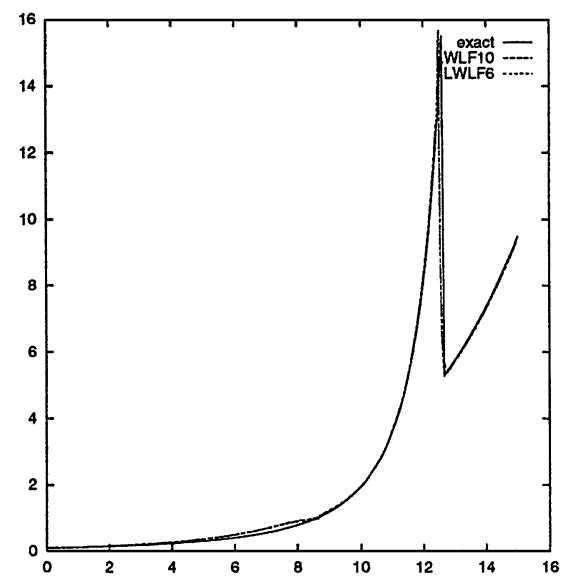

$\mathrm{b}$

FIG. 15. Exponential. (a) Exact density at initial time $t=2$ and at final time $t=6$. (b) Exact and computed density at $t=6,200$ points.

initially generated contact discontinuity no LF steps are performed in the cells adjacent to the tracked point. In addition, a Riemann solver is used to obtain the pressure and velocity at the interface, although this seems to be less important than doing only LW adjacent to the interface.

The data for the Lax problem are as follows. For the left state, $\rho=.445, p=3.528, u=$ .698 , and for the right state $\rho=.5, p=.571, u=0$. The gas is ideal with $\gamma=1.4$. The scheme is LWLF4. The results are in Fig. 17.

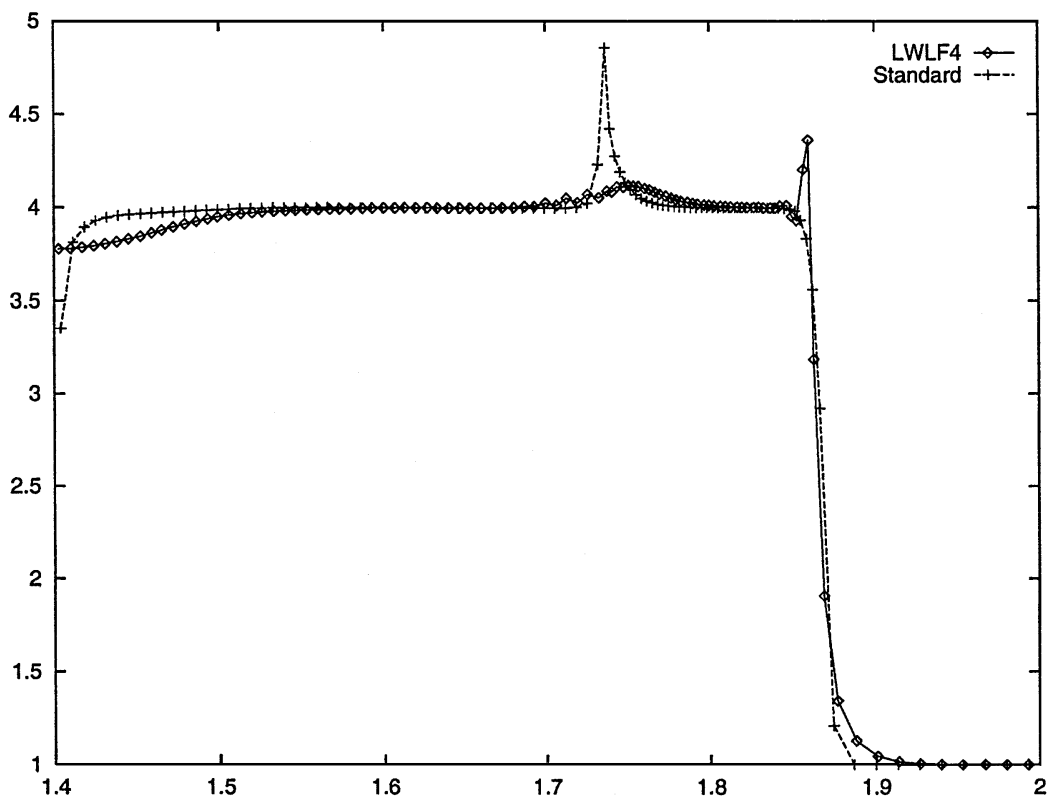

FIG. 16. Density for jump test at $t=1.4$ for standard Lagrangian method and LWLF4. 


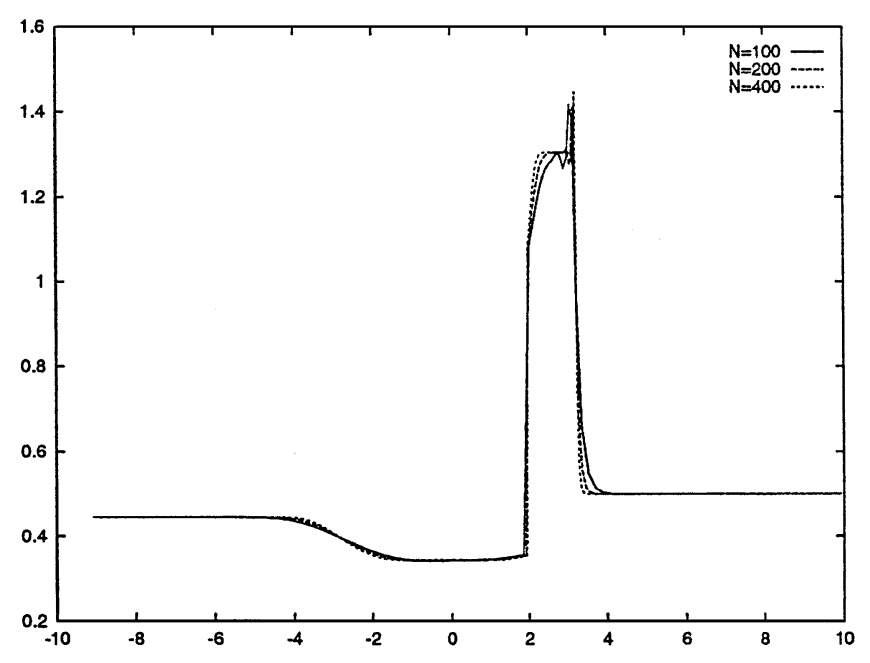

FIG. 17. Lax Riemann Problem. Convergence test.

\section{CONCLUSION}

We have computed a variety of problems in Lagrangian coordinates using the LaxFriedrichs Lax-Wendroff composite scheme LWLFn, consisting of repeated cycles composed of $n-1$ steps of a variant of Lax-Wendroff followed by one step of Lax-Friedrichs. The values of $n$ used range from four to ten, with four or six being typical. The particular values are problem dependent, but they can be chosen based on the behavior on coarse grids. The goal was to establish the feasibility of the composite idea in this situation. We believe the results reported have accomplished this. We appear to do well with the Noh problem on a Cartesian grid, which is the most natural one for this method. The Sedov problem seems to be easier, and we were able to do it on a polar grid as well. The outcome of the Saltzman problem was not as good as we would have liked. It is the Lax-Friedrichs smoothing step which seems to cause the most difficulty, and further research on other consistent smoothers is planned.

\section{ACKNOWLEDGMENTS}

This work was performed under the auspices of the US Department of Energy under contract W-7405-ENG36 and the DOE/BES Program in the Mathematical, Information, and Computational Sciences (MICS) Contract KC-07-01-01. The first author also acknowledge the support of DOE's Accelerated Strategic Computing Initiative (ASCI). The second author was supported by the CHAMMP Program of the US Department of Energy.

\section{REFERENCES}

1. E. J. Caramana and M. J. Shashkov, Elimination of artificial grid distortion and hourglass-type motions by means of Lagrangian subzonal masses and pressures, J. Comput. Phys. 42, 521 (1998).

2. E. J. Caramana, M. J. Shashkov, and P. P. Whalen, Formulations of artificial viscosity for multi-dimensional shock wave computations, J. Comput. Phys. 144, 70 (1998).

3. E. J. Caramana, D. E. Burton, M. J. Shashkov, and P. P. Whalen, The construction of compatible hydrodynamics algorithms utilizing conservation of total energy, J. Comput. Phys. 146, 227 (1998).

4. R. Courant, and K. O. Friedrichs, Supersonic Flow and Shock Waves (Interscience, New York, 1948). 
5. J. K. Dukowicz and B. J. A. Meltz, Vorticity errors in multidimensional Lagrangian codes, J. Comput. Phys. 99, 115 (1992).

6. B. Eilon, D. Gottlieb, and G. Zwas, Numerical stabilizers and computing time for second order accurate schemes, J. Comput. Phys. 9, 387 (1972).

7. R. Liska and B. Wendroff, Composite schemes for conservation laws, SIAM J. Numer. Anal. 35, 2250 (1998).

8. R. Liska and B. Wendroff, Analysis and computation with multi-layer fluid models, J. Comput. Phys. 137, 212 (1997).

9. W. F. Noh, Errors for calculations of strong shocks using an artificial viscosity and an artificial heat flux, J. Comput. Phys. 72, 78 (1978).

10. A. A. Samarskii and Yu. P. Popov, Difference Schemes of Gas Dynamics (Nauka, Moscow, 1975).

11. Ya. B. Zeldovich and Yu. P. Rayzer, Physics of Shock Waves and High-Temperature Hydrodynamic Phenomena (Academic Press, San Diego, 1967), Vol. II, p. 852. 